\title{
Searching for a DNAzyme version of the leadzyme
}

\author{
Runjhun Saran, Qingyun Chen, and Juewen Liu* \\ Department of Chemistry, Waterloo Institute for Nanotechnology \\ University of Waterloo, Waterloo, Ontario, N2L 3G1, Canada
}

Fax: 519 7460435; Tel: 5198884567 Ext. 38919

E-mail: liujw@uwaterloo.ca.

The final publication is available at Springer via http:// dx.doi.org/10.1007/s00239-015-9702-z 


\section{Abstract.}

The leadzyme refers to a small ribozyme that cleaves a RNA substrate in the presence of $\mathrm{Pb}^{2+}$. In an optimized form, the enzyme strand contains only two unpaired nucleotides. Most RNAcleaving DNAzymes are much longer. Two classical $\mathrm{Pb}^{2+}$-dependent DNAzymes, 8-17 and GR5, both contain around 15 nucleotides in the enzyme loop. This is also the size of most RNA-cleaving DNAzymes that use other metal ions for their activity. Such large enzyme loops make spectroscopic characterization difficult and so far no high resolution structural information is available for active DNAzymes. The goal of this work is to search for DNAzymes with smaller enzyme loops. A simple replacement of the ribonucleotides in the

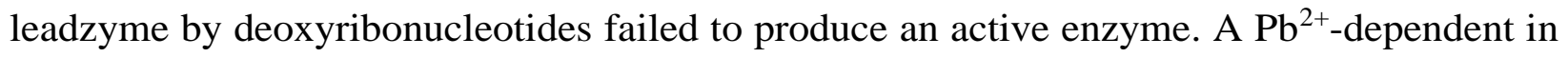
vitro selection combined with deep sequencing was then performed. After sequence alignment and DNA folding, a new DNAzyme named PbE22 was identified, which contains only 5 nucleotides in the enzyme catalytic loop. The biochemical characteristics of PbE22 were compared with those of the leadzyme and the two classical $\mathrm{Pb}^{2+}$-dependent DNAzymes. The rate of $\mathrm{PbE} 22$ rises with increase in $\mathrm{Pb}^{2+}$ concentration, being $1.7 \mathrm{~h}^{-1}$ in presence of $100 \mu \mathrm{M}$

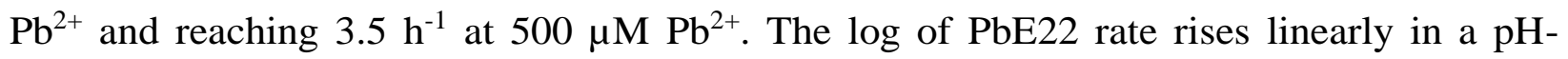
dependent fashion $\left(20 \mu \mathrm{M} \mathrm{Pb}^{2+}\right)$ with a slope of 0.74 . In addition, many other abundant sequences in the final library were studied. These sequences are quite varied in length and nucleotide composition, but some contain a few conserved nucleotides consistent with the GR5 structure. Interestingly, some sequences are active with $\mathrm{Pb}^{2+}$ but none of them were

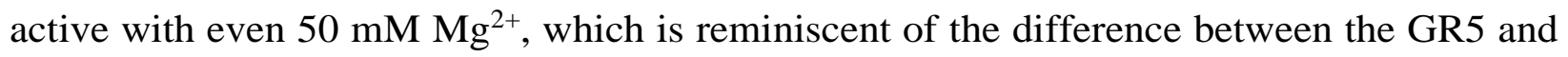
8-17 DNAzymes. 


\section{Introduction}

Most RNA-cleaving ribozymes and DNAzymes require divalent metal ions for catalysis (Lu 2002; Sigel and Pyle 2007; Ward et al. 2014). Among the different metal ions used for RNA cleavage, $\mathrm{Pb}^{2+}$ has greatly fueled the growth of this field. The interaction between $\mathrm{Pb}^{2+}$ and nucleic acids was observed more than 50 years back. In 1959, the hydrolysis of RNA by lead hydroxide was demonstrated (Dimroth et al. 1959). A detailed investigation was published in 1968 showing that the rate of RNA depolymerization as well as its $\mathrm{pH}$ optimum, both varying with the $\mathrm{Pb}^{2+}$ concentration (Farkas 1968). It first became known that $\mathrm{Pb}^{2+}$ can bring about site-specific cleavage of tRNA in 1973 (Winterme.W and Zachau 1973). The mechanism of cleavage was proposed based on biochemical data and the crystal structure of the yeast tRNA $^{\text {Phe }}$ soaked in lead acetate (Brown et al. 1985; Werner et al. 1976).

Multiple variations of this well-studied yeast tRNA were used to design RNA selection libraries to isolate RNAs that undergo autolytic cleavage in the presence of $\mathrm{Pb}^{2+}(\mathrm{Pan}$ and Uhlenbeck 1992a). Amongst the sequences derived from this selection, one was truncated and optimized into a minimal motif known as the 'leadzyme'. The leadzyme is a very small but interesting ribozyme with only two unpaired nucleotides $5^{\prime}$ rGrA3' in the enzyme loop and four unpaired nucleotides in the substrate strand (Figure 1B). This enzyme is highly specific for lead and its reaction produces a 5'-OH end along with a 2'3' cyclic phosphate product (Pan et al. 1994; Pan and Uhlenbeck 1992b). Deeper insights have been obtained about this enzyme through biochemical studies (Chartrand et al. 1997; Ohmichi et al. 1998), NMR (Hoogstraten et al. 1998; Hoogstraten et al. 2000; Legault et al. 1998), X-ray crystallography (Wedekind and McKay 1999; Wedekind and McKay 2003), and other biophysical characterizations (Kadakkuzha et al. 2009). 
Parallel to the ribozyme field, $\mathrm{Pb}^{2+}$ has been a very important metal ion in DNAzyme research as well. The first DNAzyme, GR5 (Figure 1D), was obtained as a result of a $\mathrm{Pb}^{2+}-$ dependent selection (Breaker and Joyce 1994), and it is highly specific and active with $\mathrm{Pb}^{2+}$ (Lan et al. 2010). The most extensively studied 8-17 DNAzyme, initially discovered from $\mathrm{a} \mathrm{Mg}^{2+}$-dependent selection (Santoro and Joyce 1997), is also highly active in the presence of $\mathrm{Pb}^{2+}$. A commonly used variant of the 8-17 DNAzyme is named 17E (Figure 1C) (Cruz et al. 2004; Li et al. 2000). It has been used as a model for biosensor development (Brown et al. 2003; Li and Lu 2000; Liu et al. 2009; Schlosser and Li 2010; Schlosser and Li 2009; Zhang et al. 2011).

While a few biochemical studies have been carried out on these DNAzymes, only little structure-related information is known (Brown et al. 2003; Huang and Liu 2014; Kim et al. 2007; Nowakowski et al. 1999). These two $\mathrm{Pb}^{2+}$-dependent DNAzymes (8-17 and GR5) have a similar size, containing 14 or 15 nucleotides in the enzyme loop (Figure 1C, D). This relatively large size makes it difficult to carry out X-ray crystallography or NMR studies. We reason that a shorter DNAzyme similar to the leadzyme might help in detailed spectroscopic and structural analysis. Since even two nucleotides can perform the catalytic function in the leadzyme, an interesting question is whether it is possible to achieve similar catalysis in a short DNAzyme. In this work, we employed both rational design and in vitro selection to search for very short DNAzymes. 
A

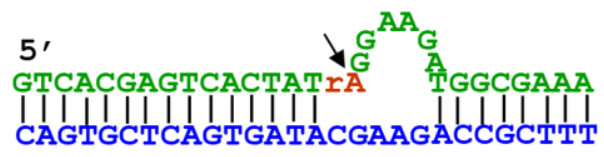

$\mathrm{PbE} 22$

C 5

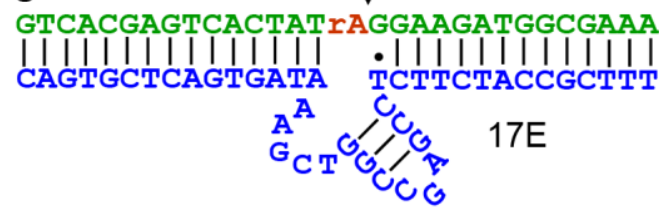

B

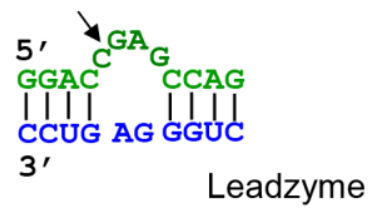

D $5^{\prime}$

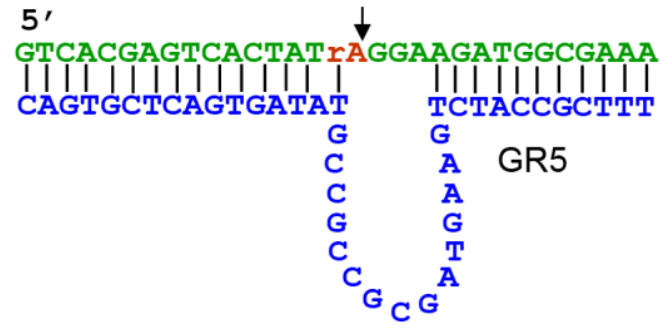

Figure 1. The secondary structures of the (A) PbE22, (B) leadzyme, (C) 17E, and (D) GR5 DNAzymes. The enzyme strands are in blue and substrate in green. The cleavage junction is indicated by the arrowheads.

\section{Materials and Methods}

Chemicals. The in vitro selection related DNA samples were purchased from Integrated DNA Technologies (IDT, Coralville, IA). For characterization, the enzyme strands were from Eurofins (Huntsville, $\mathrm{AL}) . \mathrm{Pb}(\mathrm{OAc})_{2}$ and other metal salts were from Sigma-Aldrich at the highest possible purity. Sodium acetate, 2-(N-morpholino)ethanesulfonic acid (MES), 3-(N-morpholino)propanesulfonic acid (MOPS), ethylenediaminetetraacetic acid (EDTA) disodium salt dihydrate, sodium chloride and ammonium acetate were from Mandel Scientific Inc. (Guelph, Ontario, Canada). Sso Fast EvaGreen supermix was from Bio-Radfor real-time polymerase chain reaction (PCR). T4-DNA ligase, deoxynucleotide (dNTP) mix, Taq DNA polymerase with ThermoPol buffer and low molecular weight DNA ladder were from New England Biolabs. 
In vitro Selection. The method of in vitro selection is similar to the one we reported previously (Huang et al. 2014b). In brief, for each cleavage step, the DNA library was incubated with freshly prepared $\mathrm{Pb}^{2+}$ solutions. For all the selection rounds, the metal incubation time was maintained at $60 \mathrm{~min}$ and was reduced to $5 \mathrm{~min}$ only in the last round. For all rounds, $60 \mu \mathrm{M} \mathrm{Pb}^{2+}$ was used. After incubation, the solution was mixed with $8 \mathrm{M}$ urea and purified by $10 \%$ dPAGE (denaturing polyacrylamide gel electrophoresis). The position corresponding to the cleaved product was excised from the gel, the DNA was extracted by crushing and soaking the gel and was further desalted with a Sep-Pak C18 column (Waters). After drying in an Eppendorf Vacufuge at $30{ }^{\circ} \mathrm{C}$ overnight, the dried DNA was re-suspended in $60 \mu \mathrm{L}$ of $5 \mathrm{mM}$ HEPES buffer $(\mathrm{pH} 7.5)$. The round 6 library was sent out for deep sequencing. The PCR protocols for DNA amplification were also identical to the previously published and will not be repeated here.

Gel-based activity assays. Gel-based activity assays were performed with a final concentration of $1 \mu \mathrm{M}$ of the FAM-labeled substrate strand and $2 \mu \mathrm{M}$ of the enzyme. The DNAzyme complexes were prepared by annealing them in buffer (50 mM MES, pH 6.0, $25 \mathrm{mM} \mathrm{NaCl}$ ) and a final concentration of $100 \mu \mathrm{M} \mathrm{Pb}^{2+}$ was added. For the $\mathrm{Mg}^{2+}$ assay, 50 $\mathrm{mM} \mathrm{Mg}^{2+}$ ions were added in $\mathrm{pH}$ 7.5 HEPES buffer to initiate the cleavage reaction. The products were separated on a denaturing polyacrylamide gel and analyzed using a Bio-Rad Chemi- Doc MP imaging system. For pH-dependent activity assay, the sodium acetate, 2(N-morpholino)ethanesulfonic acid (MES) and 3-(Nmorpholino) propanesulfonic acid (MOPS) buffers (50 mM buffer with $25 \mathrm{mM} \mathrm{NaCl}$ ) were used.

Deep Sequencing. The round 6 selected library was subjected to PCR and the full-length library generated from this step was used to prepare the sample for deep sequencing. The 
PCR product was subjected to another PCR reaction so that the Illumina sequencing adaptors can be added. The forward primer P701: (5'CAAGCAGAAGACGGCATACGAGATTCGCCTTAGTGACTGGAGTTCAGACGT GTGCTCTTCCGATCTCTGCAGAATTCTAATACGAGTCAC) and reverse primer P501: (5'-AATGATACGGCGACCACCGAGATCTACACTAGATCGCACACTCTTT CCCTACACGACGCTCTTCCGATCTGTGCCAAGCTTACCG), each containing a unique index sequence were used. The PCR product was purified with $2 \%$ agarose gel and extracted using a gel extraction kit from IBI Scientific. The extracted DNA was eluted in $25 \mu \mathrm{L}$ Milli-Q water and quantified using a NanoDrop Spectrophotometer.

\section{Results and Discussion}

Rational designed DNAzymes. From the study of the 8-17 and GR5 DNAzymes, it is known that both contain a few highly conserved nucleotides which are important for catalysis (Breaker and Joyce 1994; Brown et al. 2003; Peracchi et al. 2005; Schlosser et al. 2008b; Schlosser and Li 2010). In particular, the AG and CG dinucleotides in their enzyme loops have been identified to be critical. The sequence of the leadzyme, also contains the unpaired AG in the enzyme loop. Based on these, we designed a few putative DNAzyme sequences which can bind to the substrate (Figure 2A). However, when these sequences were assayed in the presence of $100 \mu \mathrm{M} \mathrm{Pb}^{2+}$ at $\mathrm{pH} 7.0$ for $2 \mathrm{~h}$, very low amount of cleavage was observed (Figure 2B). The fastest $\mathrm{Pb} 7$ has a rate of only $\sim 0.05 \mathrm{~h}^{-1}$, which is close to the background $\mathrm{RNA}$ cleavage rate by $\mathrm{Pb}^{2+}$ (vide infra) and is significantly slower than that of the leadzyme. Therefore, a simple combination of such nucleotides is insufficient for catalysis. 
A

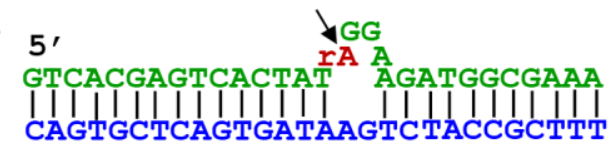

$\mathrm{Pb} 1$

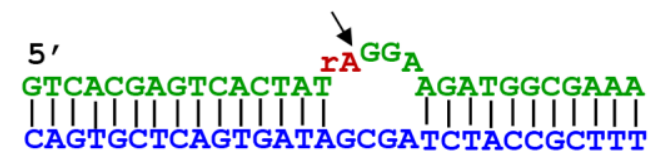

$\mathrm{Pb} 2$

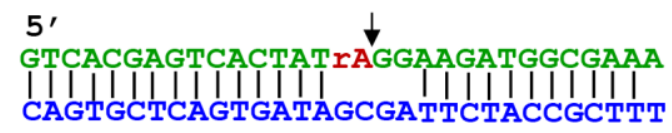

$\mathrm{Pb} 3$

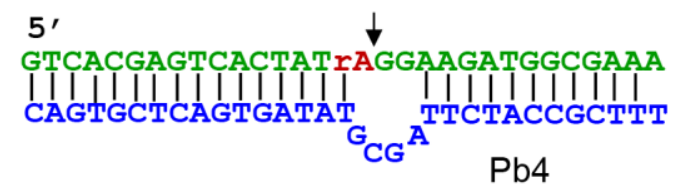

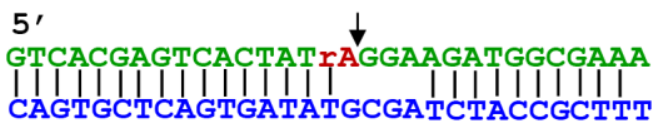

$\mathrm{Pb} 5$

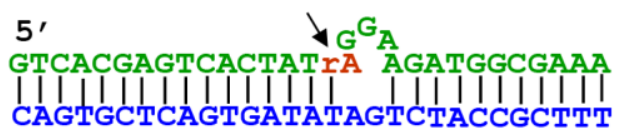

$\mathrm{Pb} 6$
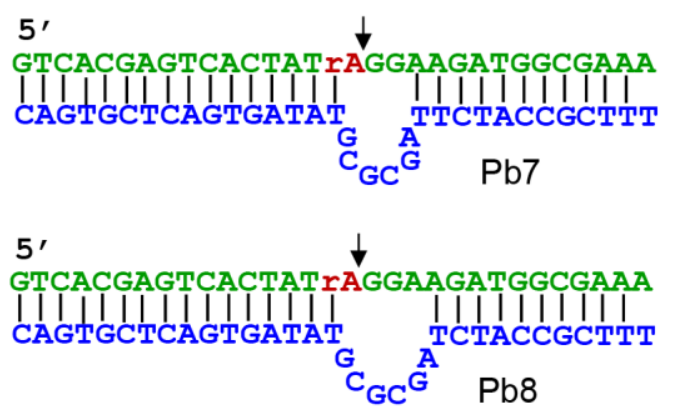

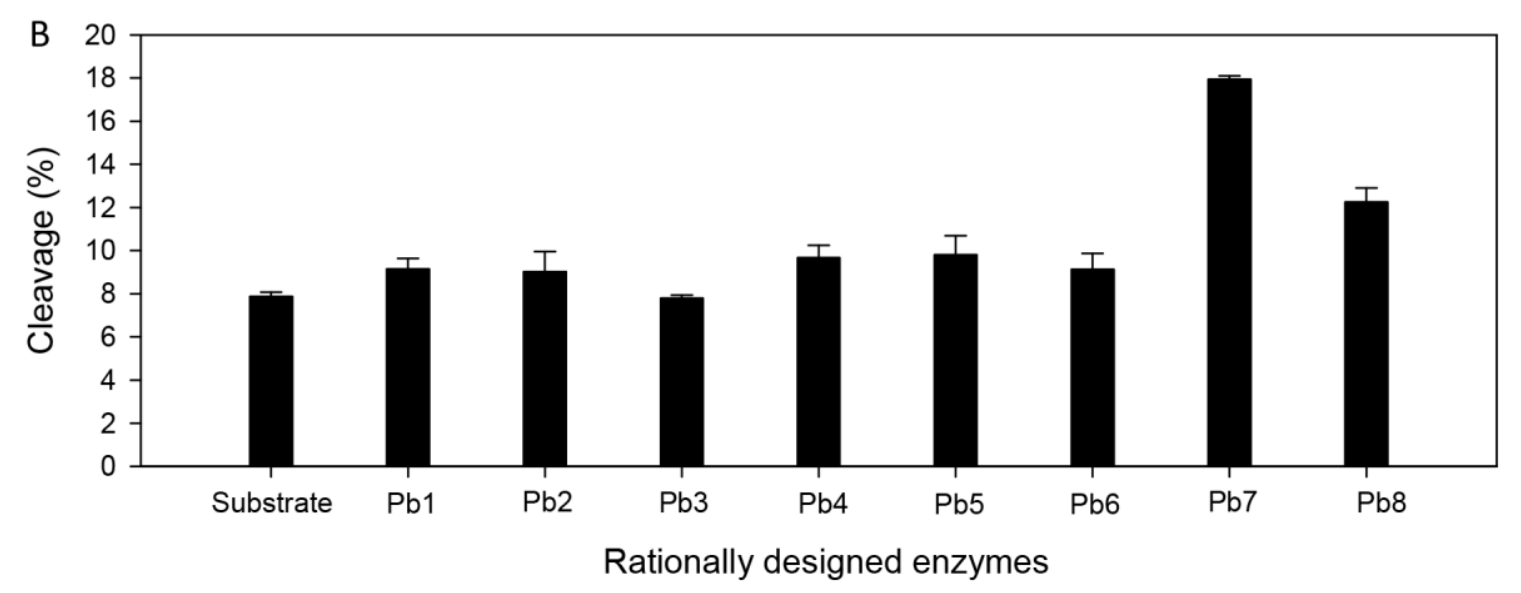

Figure 2. (A) The secondary structures of the eight rationally designed DNAzymes. (B) The cleavage yield of these enzymes in the presence of $100 \mu \mathrm{M} \mathrm{Pb}^{2+}$ at $\mathrm{pH} 7.0$ for $2 \mathrm{~h}$.

In vitro selection. Since rational design failed to produce sufficiently active DNAzymes, we next resorted to in vitro selection (Figure 3A). Since it is difficult to predict the optimal minimal length of the enzyme loop and also the number of unpaired nucleotides in the substrate strand, instead of using a very short randomized region, we employed a library 
containing 35 random nucleotides. We reason that a larger size can offer more flexibility and sequence diversity. If shorter DNAzymes exist, they may still be reflected in the final library by hiding redundant sequences as overhangs or hairpins.

A
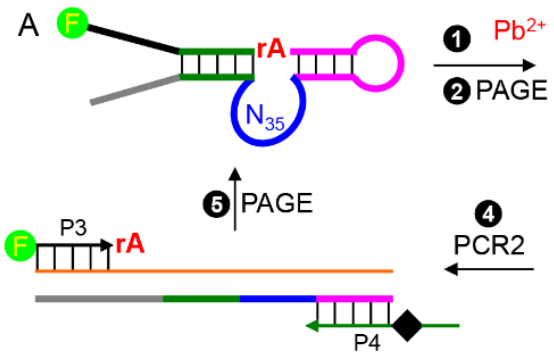

$5^{\prime}$
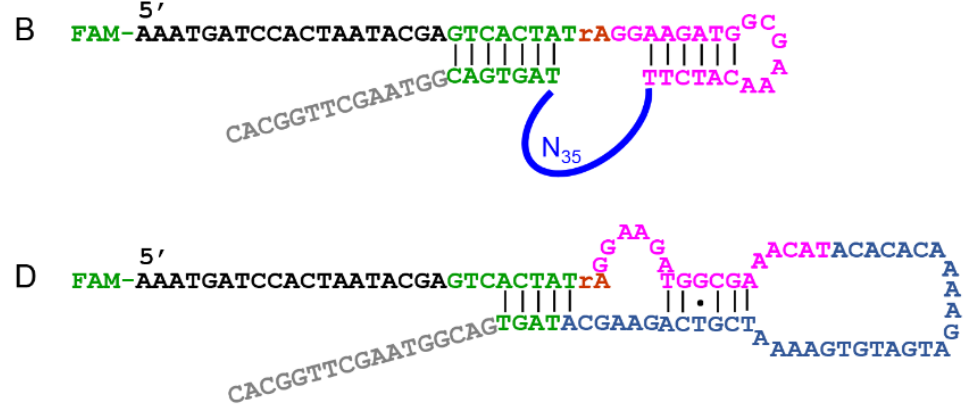

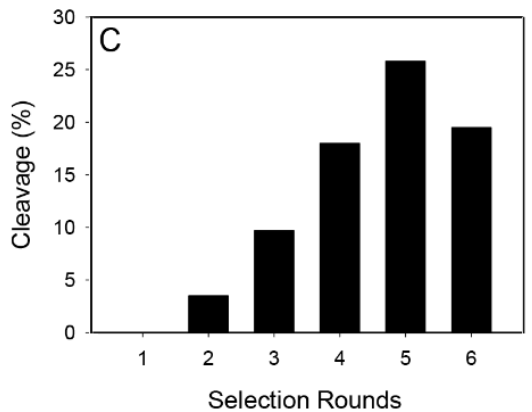

E

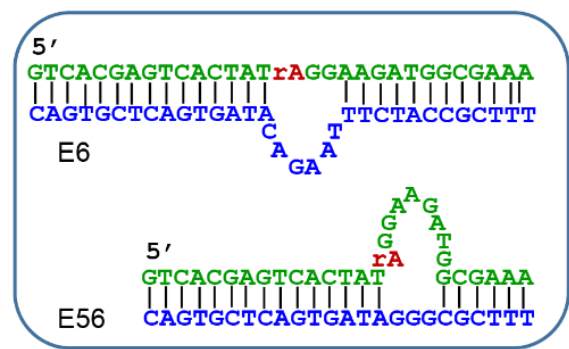

Figure 3. (A) Schematic of the in vitro selection procedure. The library contains 35 random nucleotides (N35) and a single RNA linkage (rA) serving as the cleavage site. Sequences cleaved by $\mathrm{Pb}^{2+}$ are amplified by two PCR steps to seed the next round of selection. (B) The secondary structure of the library for in vitro selection. (C) Selection progress at each round. (D) The secondary structure of the original cis-cleaving enzyme; it has been engineered to the trans-cleaving PbE22 enzyme (shown in Figure 1A). (E) Two other examples of short DNAzyme candidates from the selection, but they are inactive.

The library design is shown in Figure 3B. A single RNA linkage (rA) is embedded in this DNA library to serve as the cleavage site. Since RNA is about 1-million-fold less stable compared to DNA (Li and Breaker 1999), cleavage is most likely to take place at the RNA 
position. This library was incubated with $\mathrm{Pb}^{2+}$ and sequences that can be cleaved were separated from the rest using gel electrophoresis and amplified by two rounds of PCR to seed for the next round of selection (Figure 3A). A reaction condition of $60 \mu \mathrm{M} \mathrm{Pb}^{2+}$ for 1 $\mathrm{h}$ was pursued up to the 5 th round. The incubation time was reduced to $5 \mathrm{~min}$ in round 6 , which was then subjected to sequencing. The cleavage yield at each round is shown in Figure 3C. We did not push for very fast enzymes in this selection since we aim to obtain shorter DNAzymes, which may not cleave very efficiently. In other words, our goal was to maximize sequence diversity in the resulting library.

Instead of using conventional cloning and Sanger sequencing, we chose to use deep sequencing for this project to search for very short DNAzymes, which may not be highly active and may not represent the major population in the library. As a result, we needed to exhaustively search for all possible sequences. From the sequencing results of our final library, a total of 32,144 sequences were obtained.

Sequence analysis. It is interesting to note that while the first DNAzyme selection was carried out using $\mathrm{Pb}^{2+}, \mathrm{Pb}^{2+}$ has not been used as a metal cofactor in any subsequent selections. Therefore, this work represents a second example of such an effort. The selection condition is also quite different; while GR5 was selected using $1 \mathrm{mM} \mathrm{PbOAc}$ in a high salt buffer $(0.5 \mathrm{M} \mathrm{NaCl}, 0.5 \mathrm{M} \mathrm{KCl}, 50 \mathrm{mM}$ $\mathrm{MgCl}_{2}$ at $\mathrm{pH}$ 7) (Breaker and Joyce 1994), our selection used only $60 \mu \mathrm{M} \mathrm{Pb}^{2+}$ in a low salt buffer (50mM MES pH 6.0,25mM NaCl). Instead of only 20 sequences reported in the previous paper, we have obtained over thirty thousand sequences.

The sequenced library was aligned into different families based on their sequence similarity, and we observed quite high sequence diversity. Even the most abundant family of DNAzyme 
represents only $6.79 \%$ of the final sequences. The sequences from the first ninety families $(76.4 \%$ of the total sequences) were individually folded using Mfold (Zuker 2003). Out of these, 32 of the resulting trans-cleaving enzymes display a reasonable fold, and their trans-cleaving sequences are shown in Figure 4A. These sequences represent $46.3 \%$ of the ninety families analyzed and $35.4 \%$ of the total sequences. Some of them have the bases 5'--AGCG--CG--3' conserved exactly as they are in GR5, while a few have nucleotide insertions, mutations, or deletions from these conserved ones. Each of these 32 sequences were tested with $10 \mu \mathrm{M} \mathrm{Pb}^{2+}$ and $50 \mathrm{mM} \mathrm{Mg}^{2+}$ respectively. Their cleavage fraction after $1 \mathrm{~h}$ is plotted in Figure 4B. Interestingly, like GR5, cleavage was observed only with $\mathrm{Pb}^{2+}$; while unlike the 8-17 DNAzyme, none of them was active with $\mathrm{Mg}^{2+}$.

A

FAMILY

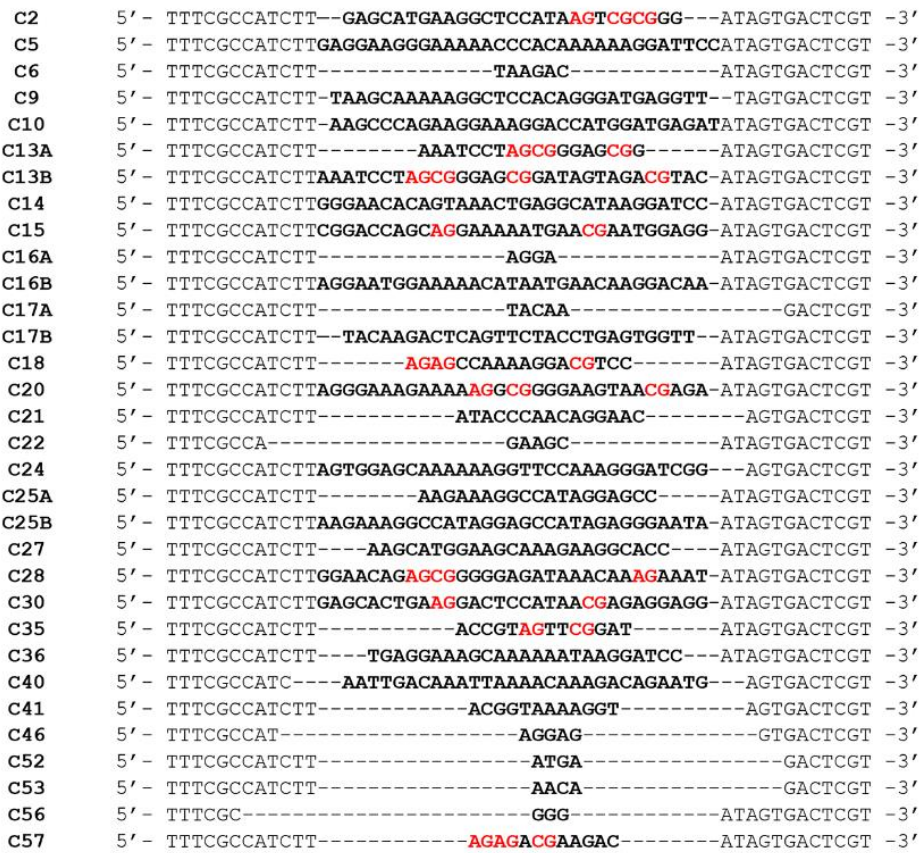

B Cleavage (\%)

\% IN FINAL LIB.

\begin{tabular}{l}
6.21 \\
4.43 \\
2.97 \\
1.96 \\
1.94 \\
1.58 \\
1.58 \\
1.49 \\
1.34 \\
1.19 \\
1.19 \\
1.12 \\
1.12 \\
1.07 \\
0.97 \\
0.92 \\
0.89 \\
0.80 \\
0.72 \\
0.72 \\
0.72 \\
0.71 \\
0.65 \\
0.59 \\
0.58 \\
0.46 \\
0.44 \\
0.40 \\
0.33 \\
0.33 \\
0.31 \\
0.28 \\
\hline
\end{tabular}

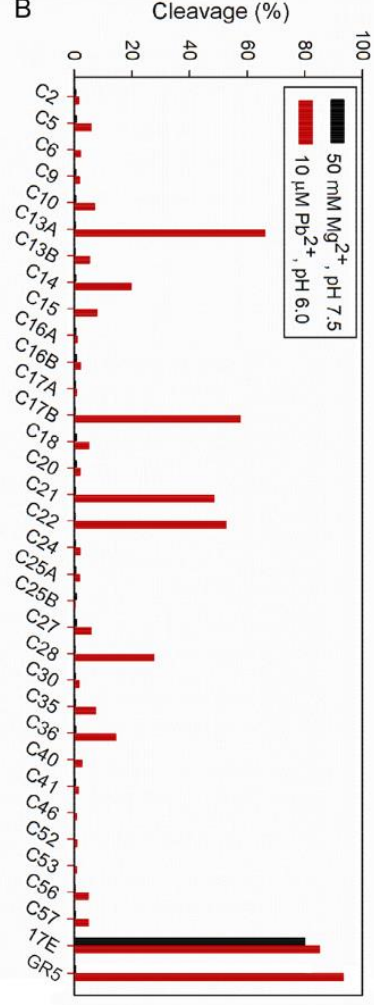

Figure 4. (A) Sequences of 32 tested DNAzymes from the selected library and their abundance in the library. The nucleotides that can be aligned with the conserved nucleotides in GR5 are marked 
in red. (B) The cleavage fraction after $1 \mathrm{~h}$ of reaction for the 32 trans-cleaving enzymes, tested with $10 \mu \mathrm{M} \mathrm{Pb}^{2+}$ (red) and $50 \mathrm{mM} \mathrm{Mg}{ }^{2+}$ (black). The 8-17 and GR5 DNAzymes are also included for comparison.

PbE22. Since the goal of this work is to identify very short DNAzymes, after the general understanding of the activity of all representing sequences, we focused our attention to very short enzyme loops. Out of the many sequences, we found only one short enzyme (family C22 in Figure 4) that exhibited decent activity, achieving $~ 50 \%$ cleavage in the above assay. This enzyme motif has appeared 285 times out of the 32,144 sequences. The truncation of this enzyme from its cis-cleaving form is shown in Figure 3D, and the transcleaving construct is shown in Figure 1A, which was re-named to be $\mathrm{PbE} 22$. PbE22 has 5 nucleotides 5'GAAGC3' in the catalytic loop of the enzyme and 6 unpaired nucleotides in the substrate strand 5'rAGGAAGA3' including the cleavage dinucleotide junction. It is interesting to note that most of these unpaired nucleotides are purines. Aside from $\mathrm{PbE} 22$, two other sequences can also fold into a short enzyme loop structure (Figure 3E). However, they are inactive when tested with $\mathrm{Pb}^{2+}$. Therefore, $\mathrm{PbE} 22$ was used for the subsequent studies.

Biochemical characterization of PbE22. To characterize this new DNAzyme, we next performed preliminary biochemical studies. First, the cleavage kinetics of PbE22 was measured in the presence of $100 \mu \mathrm{M} \mathrm{Pb}^{2+}$ (Figure 5A, black dots). The time-dependent cleavage yield can be fitted to first-order reaction kinetics with a rate constant of $1.7 \mathrm{~h}^{-1}$. Under the same condition, the free substrate was cleaved at a rate of $\sim 0.0082 \mathrm{~h}^{-1}$. Therefore, 
the rate enhancement brought by $\mathrm{PbE} 22$ in the presence of $100 \mu \mathrm{M} \mathrm{Pb}^{2+}$ at $\mathrm{pH} 7.0$ is $\sim 210-$ fold. For comparison, the GR5 DNAzyme (Figure 1D) has a reported rate enhancement of $\sim 10^{5}$ (Breaker and Joyce 1994), while the leadzyme has a rate enhancement of 1100 (Pan and Uhlenbeck 1992b). Therefore, PbE22 has the lowest catalytic efficiency, and GR5 has the highest.

The inset of Figure 5B is a gel image showing the cleavage yield at 30 min with increasing $\mathrm{Pb}^{2+}$ concentrations. Indeed more $\mathrm{Pb}^{2+}$ induced more cleavage, confirming this is a $\mathrm{Pb}^{2+}$ dependent DNAzyme. To quantitatively understand the effect of $\mathrm{Pb}^{2+}$ concentration, we next measured the enzyme kinetics at various $\mathrm{Pb}^{2+}$ concentrations at $\mathrm{pH} 7.0$ (Figure 5B). An apparent dissociation constant $\left(K_{\mathrm{d}}\right)$ of $77 \mu \mathrm{M} \mathrm{Pb}^{2+}$ was obtained. For comparison, the leadzyme has a rate of $0.4-0.5 \mathrm{~min}^{-1}$ at $25 \mu \mathrm{M} \mathrm{Pb}^{2+}$ and $\mathrm{pH}$ 7.0. This is faster than $\mathrm{PbE} 22$ but less as compared to GR5 (Pan et al. 1994). This comparison suggests that a bigger catalytic loop might be required for optimum activity of the enzyme.

pH- dependent activity. To further characterize this enzyme, we studied the DNAzyme rate with increasing $\mathrm{pH}$ at $20 \mu \mathrm{M} \mathrm{Pb}^{2+}$ concentration. Again we compared it to that of the GR5 using $1 \mu \mathrm{M} \mathrm{Pb}^{2+}$. The log of rate increased linearly with increasing $\mathrm{pH}$ in the low $\mathrm{pH}$ region with a slope of 0.74 (Figure $5 \mathrm{C}$ ). Beyond $\mathrm{pH} 7$, the increase in rate slowed down. The slope of GR5 was calculated to be 0.82 and it maintained a good linearity up to $\mathrm{pH} 7.6$ (Figure 5D). Beyond $\mathrm{pH} 7.6$, we could not measure the rate since it was too fast for manual pipetting. The reason for the narrower linear range for PbE22 might be related to the use of higher $\mathrm{Pb}^{2+}$ concentration $(20 \mu \mathrm{M})$, as $\mathrm{Pb}^{2+}$ tends to precipitate more easily at such a high concentration. Similar to PbE22, the leadzyme also exhibits a linear increase in log (rate) 
with increase in $\mathrm{pH}$ up to 7.0 at $25 \mu \mathrm{M} \mathrm{Pb}^{2+} .{ }^{11}$ Therefore, this indicates all these enzymes have a similar mechanism in terms of a single deprotonation at the rate-limiting step of this reaction, and this is often directly or indirectly linked to the deprotonation of the 2 '-OH at the cleavage site.
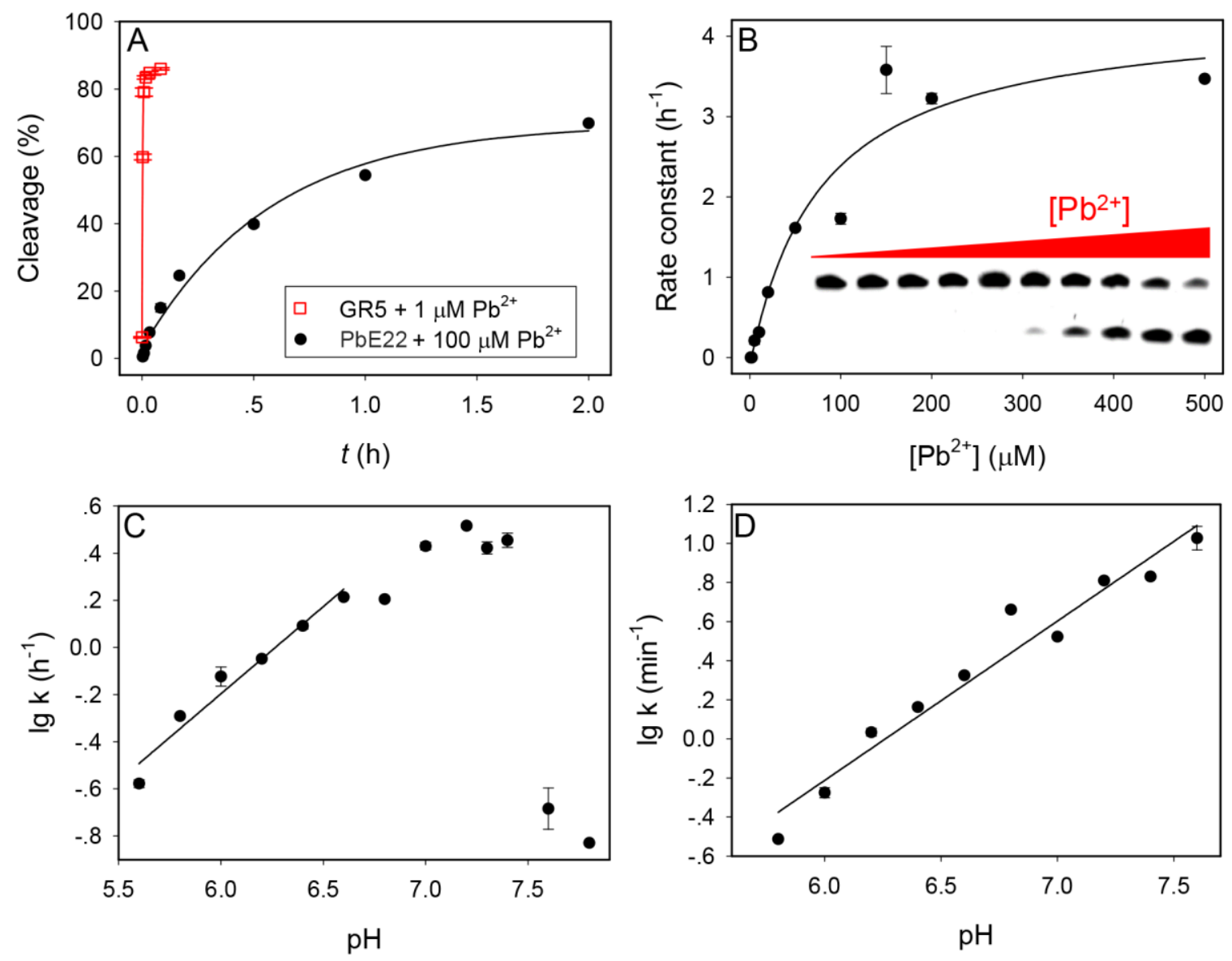

Figure 5. Biochemical characterization of PbE22. (A) Kinetics of PbE22 and GR5 cleavage at $\mathrm{pH}$ 7. (B) Cleavage rate constant of $\mathrm{PbE} 22$ as a function of $\left[\mathrm{Pb}^{2+}\right]$ at $\mathrm{pH}$ 7.0. Inset $=$ gel showing cleavage at various $\left[\mathrm{Pb}^{2+}\right]$ after 30 min reaction at $\mathrm{pH}$ 6.5. Log scale plot of the rate as a function of $\mathrm{pH}$ for (C) $\mathrm{PbE} 22$ with $20 \mu \mathrm{M} \mathrm{Pb}^{2+}$ and (D) GR5 with $1 \mu \mathrm{M} \mathrm{Pb}^{2+}$. 
Metal specificity test. GR5 has excellent selectivity for $\mathrm{Pb}^{2+}$, and an impressive fact is that GR5 is inactive even with $50 \mathrm{mM} \mathrm{Mg}{ }^{2+}$. For comparison, the 8-17 DNAzyme is quite active with such a high concentration of $\mathrm{Mg}^{2+}\left(\sim 1.6 \mathrm{~min}^{-1}\right)$ (Brown et al. 2003; Santoro and Joyce 1997; Wang et al. 2010). We also carried out metal specificity tests on PbE22. We first studied the cleavage of the substrate in presence of an array of divalent and trivalent metal ions (10 $\mu \mathrm{M}$ each, Figure 6A) and found that like GR5, PbE22 too has a high selectivity for $\mathrm{Pb}^{2+}$. Furthermore, even with $500 \mu \mathrm{M}$ of other metals still no observable activity was found (Figure 6B). Therefore, $\mathrm{PbE} 22$ is also highly specific for $\mathrm{Pb}^{2+}$.

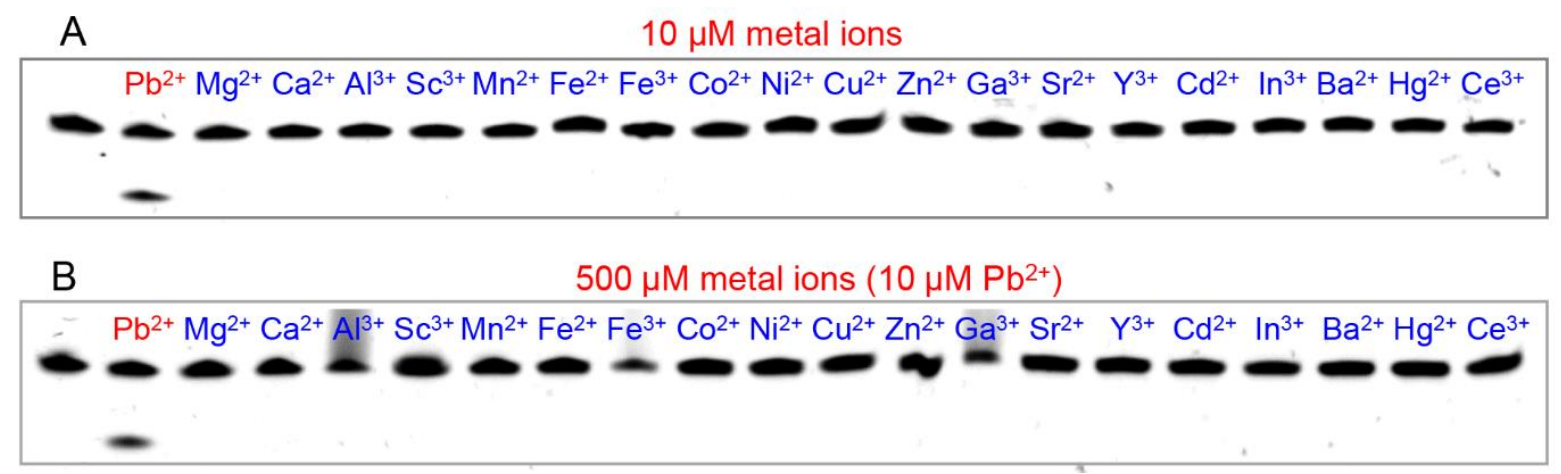

Figure 6. Metal specificity test of the PbE22 DNAzyme in the presence of (A) $10 \mu \mathrm{M}$ and (B) $500 \mu \mathrm{M}$ of all the metals compared to $10 \mu \mathrm{M}$ of $\mathrm{Pb}^{2+}$ at $\mathrm{pH} 6.2$.

It is worth mentioning here that we tested up to $0.5 \mathrm{mM} \mathrm{Pb}^{2+}$ and $50 \mathrm{mM} \mathrm{Mg}^{2+}$ for PbE22. Based on a rough estimation, at the same metal concentration, the rate of $\mathrm{Pb}^{2+}$ is $\sim 33800$ fold faster than that of $\mathrm{Mg}^{2+}$ for cleaving the 17E DNAzyme (Brown et al. 2003). This difference is even larger for the GR5 DNAzyme. Since our PbE22 is much slower, it would require over $1 \mathrm{M}$ of $\mathrm{Mg}^{2+}$ to conclude that $\mathrm{PbE} 22$ is inactive with $\mathrm{Mg}^{2+}$ if we use $17 \mathrm{E}$ as the standard. We tested the PbE22 in $4 \mathrm{M} \mathrm{Mg}^{2+}$ and found that it has no cleavage as well (data not shown). Therefore, $\mathrm{PbE} 22$ is highly specific for $\mathrm{Pb}^{2+}$. 
Further discussion. The PbE22 DNAzyme gives an example for better understanding of the effect of the size of the catalytic loop on the activity of DNAzyme. This study indicates that although site-specific and metal-specific catalysis is possible with short loops, the presence of extra nucleotides is probably needed for optimal activity. It suggests the significance of bigger catalytic loops for better folding or scaffolding for utilizing the metal cofactor. For example, in the 8-17 DNAzyme, in addition to the four nucleotides identified to be critical for the cleavage reaction, additional nucleotides are found to have other roles to assist DNAzyme folding (Schlosser et al. 2008b; Wang et al. 2010). In this PbE22 DNAzyme, the number of nucleotides on the enzyme strand is very limited. At the same time, DNA lacks the structural versatility present in RNA due to the lack of the $2^{\prime}-\mathrm{OH}$ group, which may explain the faster cleavage by the leadzyme despite its even smaller size. Currently, most DNAzyme selections use 30 to 50 random nucleotides in the selection library. When the catalytic core is examined, however, the required sequences are much shorter. Usually the core contains $\sim 15$ nucleotides for RNA-cleaving DNAzymes. To deal with the remaining nucleotides, one of the common strategies adopted by the enzymes is to form a hairpin. This hairpin may or may not play an important role in catalysis. The DNAzyme folding may turn out to be very different if no hairpin is added or deleted and this may affect DNAzyme activity. For example, in the $\mathrm{UO}_{2}{ }^{2+}$ specific DNAzyme, removal of the hairpin completely suppresses its activity (Brown et al. 2009; Liu et al. 2007). On the other hand, for the lanthanide-specific DNAzyme Ce13d, removal of the hairpin has relatively less effect (Huang et al. 2014a). Another strategy to deal with the redundant nucleotides is by forming alternative substrate binding regions (e.g. a portion of the library is used for the substrate binding purpose). 
Given the ability of longer libraries to hide extra bases, we decided to use the N35 library in this work. In addition, it may allow more flexibility in substrate binding than directly using very short libraries. For example, some nucleotides nearby the cleavage site may need to be non-paired, and this is particularly true for the short enzymes (the leadzyme and PbE22 are the examples). A large library may better support such possibility by forming alternative base pairing with the substrate. Finally, since we cannot predict the minimal length to use, we counted on the sequence diversity and flexibility of the long library to obtain short DNAzymes in a single selection experiment. The successful isolation of the PbE22 DNAzyme has indicated the success and reliability of this strategy. There have been studies using a shorter random region of N20 for carrying out DNAzyme selections (Schlosser et al. 2008a). One of the future directions is to test even smaller randomized regions and this may directly eliminate GR5 like sequences.

Traditionally, at the end of in vitro selection, the selected library is cloned into plasmid vectors and transformed into the bacterial cells which are further grown and disrupted for isolating the amplified plasmids. These purified plasmids are then subjected to sequencing. Although, the method is widespread in usage, it poses certain limitations upon the number of sequences and diversity of sequences that can be obtained. As a result, only a small fraction of the selected library and mostly the most abundant sequences can be obtained. This leads to loss of valuable information regarding the selection and can prove to be a great hindrance in the discovery of new enzymes. However, the technology of deep sequencing is bringing about a revolution in this area. Through this, thousands of sequences can be obtained from the selected library which can further be subjected to clustering based on their sequence similarities. A comprehensive understanding of the diversity and abundance of each sequence family can be obtained and significant sequence variations 
within each type of family can also be dug out and analysed. This gives a huge pool of information. For example, in the above mentioned selection, PbE22 could be fished out of 32,144 sequences in spite of its abundance being only $0.89 \%$ amongst the entire pool. Thus, this demonstrates the power of the technology of deep sequencing. Several other selection studies have already explored this deep sequencing technique to reveal diverse sequence information (Ameta et al. 2014; Majerfeld et al. 2010; Mao et al. 2015; Pitt and FerréD’Amaré 2010).

The PbE22 DNAzyme is not a very efficient DNAzyme, but it may provide a scaffold for studying $\mathrm{Pb}^{2+}$ binding to DNAzyme given its much smaller size. Subsequent work will be focused on testing its structure and spectroscopic properties.

\section{Conclusions}

In summary, we had a goal of obtaining a very short RNA-cleaving DNAzyme. To achieve this goal, both rational design and in vitro selection were performed. We isolated a very short RNAcleaving DNAzyme that uses $\mathrm{Pb}^{2+}$ as a cofactor named $\mathrm{PbE} 22$. It has a rate enhancement of $\sim 200-$ fold and this is lower compared to that for the leadzyme or the GR5 DNAzyme. These enzymes shares a similar cleavage mechanism as deduced from $\mathrm{pH}$-dependent studies. PbE22 has excellent selectivity for $\mathrm{Pb}^{2+}$. In the rest of the library too, all the active sequences are only active with $\mathrm{Pb}^{2+}$ but not with $\mathrm{Mg}^{2+}$. This study has provided insights into the significance of the size of the

DNAzymes, $\mathrm{Pb}^{2+}$-dependent activity and metal specificity. We expect that this DNAzyme can be used for spectroscopic and structural analysis. These experiments have been difficult to carry out with the current DNAzymes that bear relatively large catalytic loops. 


\section{Acknowledgement}

Funding for this work was from the University of Waterloo, Ontario Ministry of Research \& Innovation, and the Natural Sciences and Engineering Research Council (NSERC) of Canada (Discovery Grant and Strategic Project Grant).

\section{References}

Ameta S, Winz M-L, Previti C, Jäschke A (2014) Next-generation sequencing reveals how RNA catalysts evolve from random space. Nucleic Acids Res. 42:1303-1310

Breaker RR, Joyce GF (1994) A DNA enzyme that cleaves RNA. Chem. Biol. 1:223-229

Brown AK, Li J, Pavot CMB, Lu Y (2003) A lead-dependent DNAzyme with a two-step mechanism. Biochemistry 42:7152-7161

Brown AK, Liu JW, He Y, Lu Y (2009) Biochemical characterization of a uranyl ion-specific DNAzyme. ChemBioChem 10:486-492

Brown RS, Dewan JC, Klug A (1985) Crystallographic and biochemical investigation of the lead(II)-catalyzed hydrolysis of yeast phenylalanine tRNA. Biochemistry 24:4785-4801

Chartrand P, Usman N, Cedergren R (1997) Effect of structural modifications on the activity of the leadzyme. Biochemistry 36:3145-3150

Cruz RPG, Withers JB, Li Y (2004) Dinucleotide junction cleavage versatility of 8-17 deoxyribozyme. Chem. Biol. 11:57-67

Dimroth K, Witzel H, Hulsen W, Mirbach H (1959) Uber die hydrolyse von ribonucleinsauren in gegenwart von metallhydroxyden. Annalen Der Chemie-Justus Liebig 620:94-108

Farkas WR (1968) Depolymerization of ribonucleic acid by plumbous ion. Biochimica et Biophysica Acta (BBA) - Nucleic Acids and Protein Synthesis 155:401-409 
Hoogstraten CG, Legault P, Pardi A (1998) NMR solution structure of the lead-dependent ribozyme: Evidence for dynamics in RNA catalysis. J. Mol. Biol. 284:337-350

Hoogstraten CG, Wank JR, Pardi A (2000) Active site dynamics in the lead-dependent ribozyme. Biochemistry 39:9951-9958

Huang P-JJ, Lin J, Cao J, Vazin M, Liu J (2014a) Ultrasensitive DNAzyme beacon for lanthanides and metal speciation. Anal. Chem. 86:1816-1821

Huang P-JJ, Liu J (2014) Two $\mathrm{Pb}^{2+}$-specific dnazymes with opposite trends in split-site-dependent activity. Chem. Commun. 50:4442-4444

Huang P-JJ, Vazin M, Liu J (2014b) In vitro selection of a new lanthanide-dependent DNAzyme for ratiometric sensing lanthanides. Anal. Chem. 86:9993-9999

Kadakkuzha BM, Zhao L, Xia T (2009) Conformational distribution and ultrafast base dynamics of leadzyme. Biochemistry 48:3807-3809

Kim HK, Rasnik I, Liu JW, Ha TJ, Lu Y (2007) Dissecting metal ion-dependent folding and catalysis of a single DNAzyme. Nat. Chem. Biol. 3:762-768

Lan T, Furuya K, Lu Y (2010) A highly selective lead sensor based on a classic lead DNAzyme. Chem. Commun. 46:3896-3898

Legault P, Hoogstraten CG, Metlitzky E, Pardi A (1998) Order, dynamics and metal-binding in the lead-dependent ribozyme. J.Mol.Biol. 284:325-335

Li J, Lu Y (2000) A highly sensitive and selective catalytic DNA biosensor for lead ions. J. Am. Chem. Soc. 122:10466-10467

Li J, Zheng W, Kwon AH, Lu Y (2000) In vitro selection and characterization of a highly efficient Zn(II)-dependent RNA-cleaving deoxyribozyme. Nucleic Acids Res. 28:481-488 
Li Y, Breaker RR (1999) Kinetics of RNA degradation by specific base catalysis of transesterification involving the 2'-hydroxyl group. J. Am. Chem. Soc. 121:5364-5372

Liu J, Brown AK, Meng X, Cropek DM, Istok JD, Watson DB, Lu Y (2007) A catalytic beacon sensor for uranium with parts-per-trillion sensitivity and millionfold selectivity. Proc. Natl. Acad. Sci. U.S.A. 104:2056-2061

Liu J, Cao Z, Lu Y (2009) Functional nucleic acid sensors. Chem. Rev. 109:1948-1998

Lu Y (2002) New transition metal-dependent DNAzymes as efficient endonucleases and as selective metal biosensors. Chem. Eur. J. 8:4588-4596

Majerfeld I, Chocholousova J, Malaiya V, Widmann J, McDonald D, Reeder J, Iyer M, Illangasekare M, Yarus M, Knight R (2010) Nucleotides that are essential but not conserved; a sufficient 1-tryptophan site in RNA. RNA 16:1915-1924

Mao Y, Liu M, Tram K, Gu J, Salena BJ, Jiang Y, Li Y (2015) Optimal DNA templates for rolling circle amplification revealed by in vitro selection. Chem. Eur. J 21:8069-8074

Nowakowski J, Shim PJ, Prasad GS, Stout CD, Joyce GF (1999) Crystal structure of an 82nucleotide RNA-DNA complex formed by the 10-23 DNA enzyme. Nat. Struct. Biol. 6:151-156

Ohmichi T, Okumoto Y, Sugimoto N (1998) Effect of substrate RNA sequence on the cleavage reaction by a short ribozyme. Nucleic Acids Res. 26:5655-5661

Pan T, Dichtl B, Uhlenbeck OC (1994) Properties of an in vitro selected $\mathrm{Pb}^{2+}$ cleavage motif. Biochemistry 33:9561-9565

Pan T, Uhlenbeck OC (1992a) In vitro selection of rnas that undergo autolytic cleavage with $\mathrm{Pb}(\mathrm{II})$. Biochemistry 31:3887-3895 
Pan T, Uhlenbeck OC (1992b) A small metalloribozyme with a two-step mechanism. Nature $358: 560-563$

Peracchi A, Bonaccio M, Clerici M (2005) A mutational analysis of the 8-17 deoxyribozyme core. J. Mol. Biol. 352:783-794

Pitt JN, Ferré-D’Amaré AR (2010) Rapid construction of empirical RNA fitness landscapes. Science 330:376-379

Santoro SW, Joyce GF (1997) A general purpose RNA-cleaving DNA enzyme. Proc. Natl. Acad. Sci. U.S.A. 94:4262-4266

Schlosser K, Gu J, Lam JCF, Li YF (2008a) In vitro selection of small RNA-cleaving deoxyribozymes that cleave pyrimidine-pyrimidine junctions. Nucleic Acids Res. 36:47684777

Schlosser K, Gu J, Sule L, Li YF (2008b) Sequence-function relationships provide new insight into the cleavage site selectivity of the 8-17 RNA-cleaving deoxyribozyme. Nucleic Acids Res. 36:1472-1481

Schlosser K, Li Y (2010) A versatile endoribonuclease mimic made of DNA: Characteristics and applications of the 8-17 RNA-cleaving DNAzyme. ChemBioChem 11:866-879

Schlosser K, Li YF (2009) Biologically inspired synthetic enzymes made from DNA. Chem. Biol. 16:311-322

Sigel RKO, Pyle AM (2007) Alternative roles for metal ions in enzyme catalysis and the implications for ribozyme chemistry. Chem. Rev. 107:97

Wang B, Cao L, Chiuman W, Li Y, Xi Z (2010) Probing the function of nucleotides in the catalytic cores of the 8-17 and 10-23 dnazymes by abasic nucleotide and C3 spacer substitutions. Biochemistry 49:7553-7562 
Ward WL, Plakos K, DeRose VJ (2014) Nucleic acid catalysis: Metals, nucleobases, and other cofactors. Chem. Rev. 114:4318-4342

Wedekind JE, McKay DB (1999) Crystal structure of a lead-dependent ribozyme revealing metal binding sites revelant to catalysis. Nat. Struct. Biol. 6:261-268

Wedekind JE, McKay DB (2003) Crystal structure of the leadzyme at 1.8 Å resolution: Metal ion binding and the implications for catalytic mechanism and allo site ion regulation. Biochemistry 42:9554-9563

Werner C, Krebs B, Keith G, Dirheimer G (1976) Specific cleavages of pure tRNAs by plumbous ions. Biochim. Biophys. Acta 432:161-175

Winterme.W, Zachau HG (1973) $\mathrm{Mg}^{2+}$-catalyzed specific splitting of transfer-RNA. Biochim. Biophys. Acta 299:82-90

Zhang X-B, Kong R-M, Lu Y (2011) Metal ion sensors based on DNAzymes and related DNA molecules. Annu. Rev. Anal. Chem. 4:105-128

Zuker M (2003) Mfold web server for nucleic acid folding and hybridization prediction. Nucleic Acids Res. 31:3406-3415 